\title{
A tiny facial pigmented macule: overcoming the diagnostic challenge
}

\author{
Athanasios J. Stefanis ${ }^{1}$, Zoe Apalla ${ }^{2}$, Chryssoula Papageorgiou ${ }^{2}$, Dimitrios Ioannides ${ }^{2}$, \\ Christina Nikolaidou ${ }^{3}$, Aimilios Lallas ${ }^{2}$
}

\begin{abstract}
1 Department of Dermatology, Third Faculty of Medicine, Charles University, Prague, Czech Republic 2 First Department of Dermatology, Aristotle University, Thessaloniki, Greece

3 Department of Histopathology, Hippokration General Hospital, Thessaloniki, Greece
\end{abstract}

Key words: dermoscopy, lentigo maligna, melanoma, pigmented actinic keratosis, solar lentigo

Citation: Stefanis AJ, Apalla Z, Papageorgiou C, Ioannides D, Nikolaidou C, Lallas A. A tiny facial pigmented macule: overcoming the diagnostic challenge. Dermatol Pract Concept 2018;8(4):322-323. DOI: https://doi.org/10.5826/dpc.0804a15

Received: February 11, 2018; Accepted: June 7, 2018; Published: October 31, 2018

Copyright: $@ 2018$ Stefanis et al. This is an open-access article distributed under the terms of the Creative Commons Attribution License, which permits unrestricted use, distribution, and reproduction in any medium, provided the original author and source are credited.

Funding: None.

Competing interests: None.

All authors have contributed significantly to this publication.

Corresponding author: Dr. Aimilios Lallas, First Department of Dermatology, Aristotle University, 124 Delfon Street, 54643, Thessaloniki, Greece. Email: emlallas@gmail.com

\section{Introduction}

Early diagnosis of lentigo maligna (LM) represents one of the most challenging tasks in dermato-oncology. This is because pigmented actinic keratosis (pAK), solar lentigo (SL), seborrheic keratosis (SK), and lichen planus-like keratosis (LPLK) share several phenotypic features with melanoma in situ both macroscopically and on dermoscopic examination [1]. Dermoscopy remains the primary diagnostic method for differentiating among these tumors; however, currently used algorithms demonstrate a low accuracy for LM [2]. For instance, gray structures in a flat pigmented facial lesion can be a sign of LM but can be found also in pAK and LPLK [1,2]. Rhomboidal structures can be seen in both LM and pAK whereas a pseudonetwork can be additionally present in many SL and SK [1]. Total excision provides a definite diagnosis but is associated with the risk of undesirable cosmetic result.

Recently, a new algorithm was introduced with high specificity and nearly $70 \%$ sensitivity for LM. Rather than aiming to the recognition of melanoma-specific criteria, it focuses on the dermoscopic presence or absence of 7 features of nonmelanoma tumors: scales, white follicles, erythema, reticular or curved lines, structureless brown color, sharp demarcation, and classic criteria of SK such as milia-like cysts [2]. If any of these 7 features is clearly recognized and widely distributed on the surface of the lesion, the diagnosis of LM is excluded and biopsy is not necessary. In the absence of these features, the lesion is considered suspicious even without displaying any melanoma-specific criterion.

\section{Case Presentation}

We report a case of a 50-year-old woman who visited our department with a $0.8 \times 0.8 \mathrm{~cm}$ pigmented macule on the right side of her nose (Figure 1). She reported that she had the lesion for the past 10 months and did not notice any change in size, shape, or color. She reported intermittent sun exposure in the past and admitted not to use sun protection 


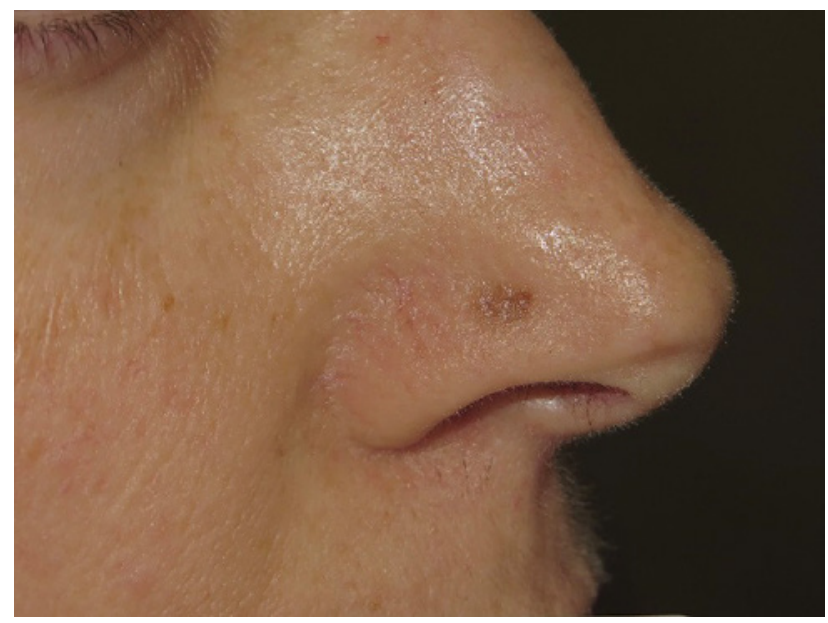

Figure 1. A flat brown macule $0.8 \times 0.8 \mathrm{~cm}$ on the right side of the nose of a 50-year-old woman. [Copyright: @2018 Stefanis et al.]

frequently. She had a negative personal and family history of melanoma or other skin cancer. Dermoscopic examination revealed a light-brown discoloration among follicular openings (pseudonetwork). No feature suggestive of melanoma could be recognized (Figure 2). In more detail, rhomboidal structures or circles were not clearly evident, follicular openings were not pigmented, gray globules could be hardly detected, and vascular network or scar-like depigmentation was not present $[3,4]$. However, neither the diagnosis of pAK nor that of SK/SL could be established, since the only detectable feature was pigmentation among the follicles (pseudonetwork). Overall, the establishment of a definite diagnosis based on clinical and dermoscopic examination was not possible.

Following the aforementioned novel algorithm, since none of the 7 nonmelanoma features was clearly present, we could not exclude the diagnosis of facial melanoma and performed an excisional biopsy. Indeed, the histopathological examination revealed melanoma in situ and the patient underwent wide excision of the scar with safety margins (Figure 3 ). In addition, the patient received adjuvant treatment with imiquimod $5 \%$ cream, consisting of 5 daily applications per week for 6 weeks [5].

\section{Conclusions}

The recognition of such a small and dermoscopically inconspicuous melanoma would be impossible by any strategy focusing on melanoma-specific structures. Early dermoscopic diagnosis of facial melanoma should rather be a diagnosis of exclusion, based on the absence of nonmelanoma features.



Figure 2. Dermoscopic examination revealed light-brown discoloration among follicular openings, which are variably sized and retain their white color (right). [Copyright: (C2018 Stefanis et al.]

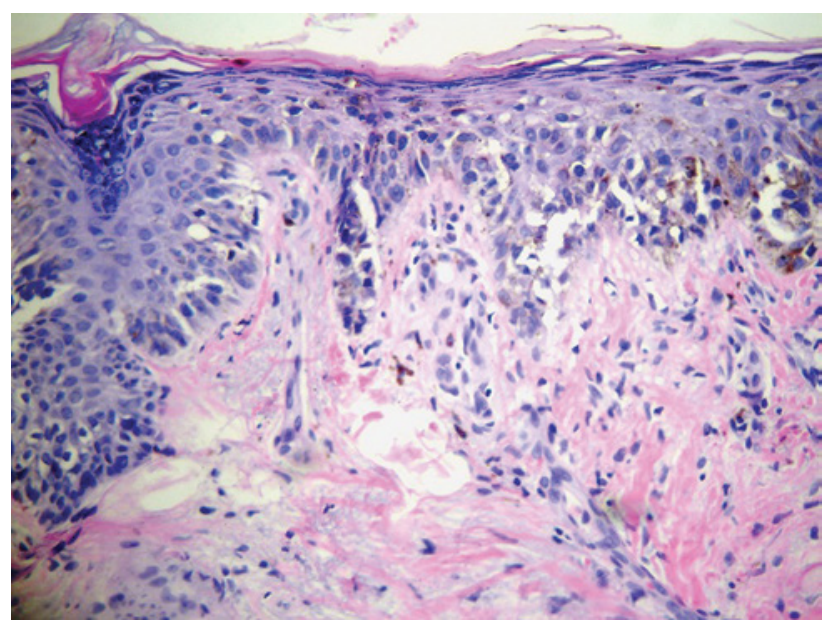

Figure 3. Photomicrograph of histology. Findings include the presence of proliferation of single atypical melanocytes along the basal cell layer and the follicular epithelium. Mild elastosis is also present. [Copyright: (C2018 Stefanis et al.]

\section{References}

1. Lallas A, Argenziano G, Moscarella E, Longo C, Simonetti V, Zalaudek I. Diagnosis and management of facial pigmented macules. Clin Dermatol. 2014;32(1):94-100.

2. Tschandl P, Gambardella A, Boespflug A, et al. Seven non-melanoma features to rule out facial melanoma. Acta Derm Venereol. 2017;97(10):1219-1224.

3. Cengiz FP, Cengiz AB, Emiroglu N, Comert E, Wellenhof RH. Dermoscopic and clinical features of head and neck melanoma. An Bras Dermatol. 2015;90(4):488-493.

4. Lallas A, Tschandl P, Kyrgidis A, et al. Dermoscopic clues to differentiate facial lentigo maligna from pigmented actinic keratosis. Br J Dermatol. 2016;174(5):1079-1085.

5. Ellis LZ, Cohen JL, High W, Stewart L. Melanoma in situ treated successfully using imiquimod after nonclearance with surgery: review of the literature. Dermatol Surg. 2012;38(6):937-946. 